\title{
Effect of Mean Flow on the Interaction between Turbulence and Zonal Flow
}

\author{
Ken UZAWA ${ }^{1)}$, Yasuaki KISHIMOTO ${ }^{1,2)}$ and Jiquan LI $^{3)}$ \\ 1) Graduate School of Energy Science, Kyoto University, Uji 611-0011, Japan \\ ${ }^{2)}$ Naka Fusion Research Establishment, JAEA, Naka 311-0193, Japan \\ ${ }^{3)}$ Southwestern Institute of Physics, P.O. Box 432, Chengdu, Sichuan 610041, P.R. China
}

(Received 26 January 2006 / Accepted 23 March 2006)

\begin{abstract}
The effects of an external mean flow on the generation of zonal flow in drift wave turbulence are theoretically studied in terms of a modulational instability analysis. A dispersion relation for the zonal flow instability having complex frequency $\omega_{\mathrm{q}}=\Omega_{\mathrm{q}}+\mathrm{i} \gamma_{\mathrm{q}}$ is derived, which depends on the external mean flow's amplitude $\left|\phi_{\mathrm{f}}\right|$ and radial wave number $k_{\mathrm{f}}$. As an example, we chose an ion temperature gradient (ITG) turbulence-driven zonal flow as the mean flow acting on an electron temperature gradient (ETG) turbulence-zonal flow system. The growth rate of the zonal flow $\gamma_{\mathrm{q}}$ is found to be suppressed, showing a relation $\gamma_{\mathrm{q}}=\gamma_{\mathrm{q} 0}\left(1-\alpha\left|\phi_{\mathrm{f}}\right|^{2} k_{\mathrm{f}}^{2}\right)$, where $\gamma_{\mathrm{q} 0}$ is the growth rate in the absence of mean flow and $\alpha$ is a positive numerical constant. This formula is applicable to a strong shearing regime where the zonal flow instability is stabilized at $\alpha\left|\phi_{\mathrm{f}}^{2}\right| k_{\mathrm{f}}^{2} \simeq 1$. Meanwhile, the suppression is accompanied by an increase of the real frequency $\left|\Omega_{q}\right|$. The underlying physical mechanism of the suppression is discussed.
\end{abstract}

(C) 2006 The Japan Society of Plasma Science and Nuclear Fusion Research

Keywords: drift wave turbulence, zonal flow, mean plasma flow, modulational instability

DOI: $10.1585 /$ pfr. 1.024

It is widely recognized that large-scale structures nonlinearly generated from maternal turbulence, such as zonal flows and streamers, play an important role in regulating turbulent transport in tokamak plasmas $[1,2]$. Specifically, zonal flows that show poloidally and toroidally symmetric potential fluctuation have attracted much attention since they break up turbulent vortices and suppress heat flux.

The generation mechanisms of such large scale structures have been intensively studied. The modulational process has been discussed as one of the plausible candidates of such generation mechanisms [3]. From an experimental viewpoint, it is desirable to know the parameters that may govern the modulational process and related structures in order to control the turbulent transport. Magnetic structure is one of these parameters. Namely, zonal flows generated from the electron temperature gradient (ETG)-driven turbulence [4] are found to be preferentially excited in a weak magnetic shear regime, whereas streamers are observed in an opposite strong magnetic shear regime. The geodesic acoustic mode (GAM) is effectively excited in a relatively high $q$ region [5].

Another important factor governing turbulent transport is an external sheared mean flow. Besides neoclassically driven mean flows, zonal flows driven by other scale fluctuations originating from different free energy sources may also be a candidate for such mean flows. It is generally believed that the sheared mean flow directly suppresses the transport through the decorrelation of tur-

author'se-mail:uzawa@center.iae.kyoto-u.ac.jp bulent vortices [6,7]. However, such a mean flow may also affect the generation of the zonal flow and indirectly change the turbulent transport.

Kim and Diamond investigated zonal flow generation in the presence of a sheared mean flow based on a wavekinetic equation and found that the mean shear flow suppresses the growth rate of the zonal flow [8]. However, this result is valid only within a weak shearing limit, and a strong shearing regime where the shearing rate becomes comparable to the growth rate of zonal flow has not been analyzed. Furthermore, the assumption of the scale separation which is applied in the wave kinetic equation is not always satisfied, as we see later.

In this paper, we investigate the generation of zonal flow influenced by a mean flow having arbitrary spatiotemporal scales including the strong shearing regime. For this purpose, we employed an alternative coherent mode coupling method for the modulational instability analysis, which assumes neither the explicit scale separation nor the interaction strength. As an example, we consider the generation of zonal flow due to the ETG turbulence, assuming that the mean flow originates from the ion temperature gradient (ITG)-driven zonal flow. Therefore, the radial wavelength is longer than that of the ETG turbulence as $k_{\mathrm{f}} \sim k_{x}^{(\mathrm{ITG})} \ll k_{x}$, where $k_{\mathrm{f}}, k_{x}^{(\mathrm{ITG})}$, and $k_{x}$ are typical radial wave numbers of the mean flow, the ITG, and ETG turbulence, respectively. In this case, the real frequency $\omega_{\mathrm{f}}$ may range from a low value $\omega_{\mathrm{f}} \ll \omega_{* \mathrm{i}}$ for the stationary ITG turbulence-driven zonal flow to a high value $\omega_{\mathrm{f}} \sim v_{\mathrm{i}} / q R$ 
for the GAM excitation, where $\omega_{* \mathrm{i}}, v_{\mathrm{i}}$ and $R$ are the ion diamagnetic frequency, the ion thermal velocity, and major radius, respectively. A dispersion relation for the complex eigen-frequency of the zonal flow, i.e. $\omega_{\mathrm{q}}=\Omega_{\mathrm{q}}+\mathrm{i} \gamma_{\mathrm{q}}$, in the presence of a time-varying mean flow is derived.

We also found that the external mean flow plays a role in suppressing the growth rate of the zonal flow, supporting the result reported by Kim and Diamond. Here, we extended the analysis to a strong shearing regime and found that the mean flow can stabilize the zonal flow generation in the short wavelength and/or strong amplitude regimes. The effect of the time-varying nature of the mean flow is found to be small in the frequency range of the GAM.

Here we consider a simple slab geometry, where $(x, y)$ denote the radial and poloidal directions, and $z$ is the direction of a uniform magnetic field. We assume that the ETG turbulence is modeled by a pump wave:

$$
\tilde{\phi}_{\mathrm{p}}(\boldsymbol{r}, t)=\phi_{0} \exp \left[\mathrm{i}\left(k_{x 0} x+k_{y 0} y-\omega_{0} t\right)\right]+\text { c.c. },
$$

where $\left(k_{x 0}, k_{y 0}\right)$ is the radial and poloidal wave numbers, and $\omega_{0}$ is the real frequency. Hereafter, we normalize the space coordinate, time, and potential by $\rho_{\mathrm{e}}, L_{\mathrm{n}} / v_{\mathrm{te}}$, and $\rho_{\mathrm{e}} T_{\mathrm{e}} / e L_{\mathrm{n}}$, respectively, where $\rho_{\mathrm{e}}, v_{\mathrm{te}}, L_{\mathrm{n}}$ are the electron Larmor radius, the electron thermal velocity, and the scale length of electron density. Here, the linear drift wave dispersion relation $\omega_{0}=-k_{y 0} /\left(1+k_{0}^{2}\right)$ is satisfied, where $k_{0}^{2}=k_{x 0}^{2}+k_{y 0}^{2}$. An arbitrarily small perturbation is regarded as the seed of the secondary mode

$$
\tilde{\phi}_{\mathrm{q}}(\boldsymbol{r}, t)=\phi_{\mathrm{q}} \exp \left[\mathrm{i}\left(k_{x \mathrm{q}} x+k_{y \mathrm{q}} y-\omega_{\mathrm{q}} t\right)\right]+\text { c.c. }
$$

where $\left(k_{x \mathrm{q}}, k_{y \mathrm{q}}\right)$ are the radial and poloidal wave number. From the non-linear interaction in the Hasegawa-Mima (HM) equation [9], two side-bands given by

$$
\tilde{\phi}_{ \pm}(\boldsymbol{r}, t)=\phi_{ \pm} \exp \left[\mathrm{i}\left(k_{x \pm} x+k_{y \pm} y-\omega_{ \pm} t\right)\right]+\text { c.c. }
$$

are excited from the coupling between the pump wave and the secondary wave, where the definitions $k_{x \pm}=k_{x 0} \pm$ $k_{x \mathrm{q}}, k_{y \pm}=k_{y 0} \pm k_{y \mathrm{q}}$ and $\omega_{ \pm}=\omega_{0} \pm \omega_{\mathrm{q}}$ are used. Here we introduce a mean flow given by

$$
\tilde{\phi}_{\mathrm{f}}(\boldsymbol{r}, t)=\phi_{\mathrm{f}} \exp \left[\mathrm{i}\left(k_{\mathrm{f}} x-\omega_{\mathrm{f}} t\right)\right]+\text { c.c. },
$$

where $\omega_{\mathrm{f}}, k_{\mathrm{f}}$, and $\phi_{\mathrm{f}}$ are the real angular frequency, radial wave number, and complex amplitude, respectively. Note that these physical quantities that characterize the mean flow are arbitrarily chosen as external parameters. Four secondary side bands with the wave numbers $\left(k_{x+} \pm k_{\mathrm{f}}, k_{y_{+}}\right)$ and $\left(k_{x-} \pm k_{\mathrm{f}}, k_{y-}\right)$ are then excited through the coupling between the original side bands, Eq. (3), and the mean flow, Eq. (4).

From the modulational process among above nine waves (a pump, a mean flow, a secondary wave, and six side bands), we constructed a dispersion relation for the secondary mode as follows:

$$
\omega_{\mathrm{q}}=\omega_{\mathrm{q}}\left(\boldsymbol{k}_{\mathrm{q}} ; k_{0}, \omega_{0},\left|\phi_{0}\right|^{2} ; k_{\mathrm{f}}, \omega_{\mathrm{f}},\left|\phi_{\mathrm{f}}\right|^{2}\right) .
$$

The dispersion relation is given by an eleventh-order algebraic equation with respect to the complex zonal flow frequency $\omega_{\mathrm{q}}$, and the growth rate is chosen from the eleven complex roots as a function of $\left(k_{x \mathrm{q}}, k_{y \mathrm{q}}\right)$. The explicit expression will be described in another paper.

In the absence of the mean flow $\left(\left|\phi_{\mathrm{f}}\right|^{2}=0\right)$ or in the long wavelength limit $\left(k_{\mathrm{f}}=0\right)$, Eq. (5) is reduced to the dispersion relation obtained by $\mathrm{Li}$ and Kishimoto [4],

$$
\begin{aligned}
& \omega_{\mathrm{q}}\left(1+k_{x \mathrm{q}}^{2}\right)+k_{y \mathrm{q}} \\
= & \frac{-2 k_{\mathrm{q}}^{2} \Lambda_{-}^{2}\left(k_{0}^{2}-k_{\mathrm{q}}^{2}\right)\left[\omega_{\mathrm{q}}\left(\Lambda_{0}-4 \Lambda_{+}^{2} / k_{\mathrm{q}}^{2}\right)+k_{y \mathrm{q}}\right]\left|\phi_{0}\right|^{2}}{\left(2 \omega_{0} \Lambda_{+}+k_{y \mathrm{q}}+\Lambda_{0} \omega_{\mathrm{q}}\right)^{2}-\left(\omega_{0} k_{\mathrm{q}}^{2}+2 \Lambda_{+} \omega_{\mathrm{q}}\right)^{2}},
\end{aligned}
$$

where $\Lambda_{0}=1+k_{0}^{2}+k_{\mathrm{q}}^{2}, \Lambda_{+}=k_{x \mathrm{q}} k_{x 0}+k_{y \mathrm{q}} k_{y 0}$, and $\Lambda_{-}=$ $k_{x \mathrm{q}} k_{y 0}-k_{y \mathrm{q}} k_{x 0}$ are used. Note that $\left(k_{x \mathrm{q}} \neq 0, k_{y \mathrm{q}}=0\right)$ and $\left(k_{x \mathrm{q}}=0, k_{y \mathrm{q}} \neq 0\right)$ correspond to zonal flow and streamer, respectively.

Here we consider the generation of zonal flow by choosing $k_{y \mathrm{q}}=0$. The structure of the secondary wave was found to be related to that of maternal turbulence [4]. Namely, a radially longer pump wave $\left(k_{x 0} \ll k_{y 0}\right)$ tends to excite zonal flows, whereas a poloidally longer pump wave $\left(k_{x 0} \gg k_{y 0}\right)$ tends to excite streamers. We consider the former case to efficiently excite zonal flow by choosing $k_{x 0}=$ $\sin (0.05 \pi)$ and $k_{y 0}=\cos (0.05 \pi)$. Assuming that the amplitude and wave number of the ITG turbulence-driven zonal flow are the same order as those of the ITG turbulence, the corresponding ratio of the potential and wave number between ETG turbulence and ITG turbulence-driven zonal flow are estimated as $\left|\phi_{0}^{(\mathrm{ETG})} / \phi_{\mathrm{ZF}}^{(\mathrm{ITG})}\right| \simeq k_{\mathrm{f}} / k_{0}=\sqrt{m_{\mathrm{e}} / m_{\mathrm{i}}} \simeq$ $1 / 43$. We have numerically solved Eq. (5) as a function of $\left(\omega_{\mathrm{f}}, k_{\mathrm{f}}\right)$ and $\left|\phi_{\mathrm{f}}\right|^{2}$.

In Fig. 1, the growth rate of zonal flow $\gamma_{\mathrm{q}}$ is illustrated as a function of $k_{x \mathrm{q}}$ in the cases of (a) $k_{\mathrm{f}}=0$ (equivalently the case with no mean flow) and (b) $k_{\mathrm{f}}=0.0125$ and $\left|\phi_{\mathrm{f}}\right|^{2}=2000$. It is found that the mean flow suppresses the growth rate by stabilizing the long wavelength region around $0<k_{x \mathrm{q}}<0.48$ and shrinking the instability window. The radial wave number of zonal flow that provides a maximum growth rate maintains a constant value,

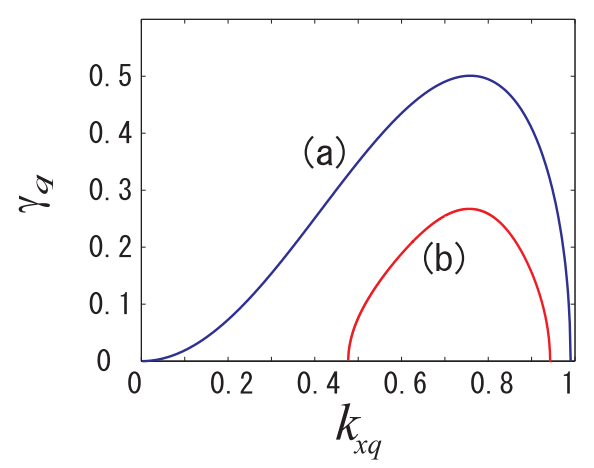

Fig. 1 The dependence of the growth rate of zonal flow as a function of the wave number of zonal flow $k_{x q}$ in the case of (a) $k_{\mathrm{f}}=0$ and (b) $k_{\mathrm{f}}=0.0125$ and $\left|\phi_{\mathrm{f}}\right|^{2}=2000$. 

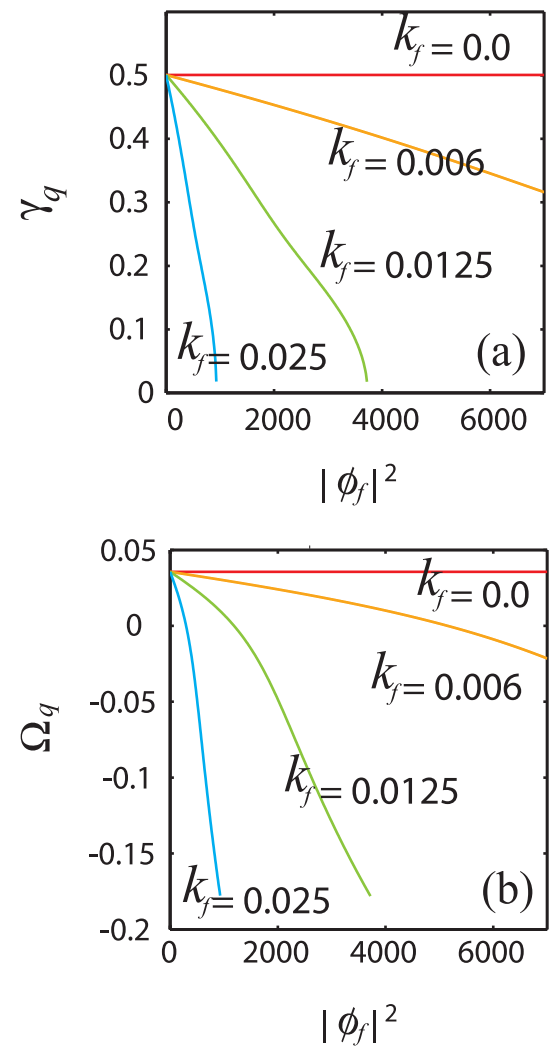

Fig. 2 The dependence of (a) growth rate $\gamma_{\mathrm{q}}$ and (b) real frequency $\Omega_{\mathrm{q}}$ as a function of mean flow energy $\left|\phi_{\mathrm{f}}\right|^{2}$. The real frequency of the mean flow is chosen to $\omega_{\mathrm{f}}=0$.

$k_{x \mathrm{q}}=0.75$, insensitive to the mean flow, which is the same order as the pump wave $k_{0}=1.0$.

Figure 2 shows the dependence of (a) growth rate $\gamma_{\mathrm{q}}$ and (b) real frequency $\Omega_{\mathrm{q}}$ of the zonal flow with respect to the mean flow energy $\left|\phi_{\mathrm{f}}\right|^{2}$ for various $k_{\mathrm{f}}$ in the case of $\omega_{\mathrm{f}}=0$ (i.e., stationary mean flow). It is found that the growth rate also shows a tendency to decrease with respect to $\left|\phi_{\mathrm{f}}\right|^{2}$. The suppression effect becomes more prominent as the wavelength of the mean flow becomes shorter as seen in Fig. 2(a). This may be compared to the $\boldsymbol{E} \times \boldsymbol{B}$ shearing suppression of vortices by an external mean flow [6,7], although the parametric dependence is different. As shown in Fig. 2(b), we note that the real frequency $\left|\Omega_{\mathrm{q}}\right|$ once crosses zero value and increases with $\left|\phi_{\mathrm{f}}\right|^{2}$ to the same order as the growth rate, suggesting that the mean flow suppresses the growth rate by having a large real frequency.

Figure 3 illustrates the contour plot of (a) growth rate $\gamma_{\mathrm{q}}$ and (b) real frequency $\Omega_{\mathrm{q}}$ in the $\left(k_{\mathrm{f}}^{2},\left|\phi_{\mathrm{f}}\right|^{2}\right)$ space. It is shown that the growth rate of the zonal flow decreases almost symmetrically with respect to $k_{\mathrm{f}}^{2}$ and $\left|\phi_{\mathrm{f}}\right|^{2}$, so that the growth rate is given approximately by

$$
\gamma_{\mathrm{q}} \simeq \gamma_{\mathrm{q} 0}\left(1-\alpha\left|\phi_{\mathrm{f}}\right|^{2} k_{\mathrm{f}}^{2}\right)
$$

where $\gamma_{\mathrm{q} 0}$ is the growth rate without mean flow, and $\alpha$ is a numerical constant roughly given by $\alpha=1.2-1.8$ in the
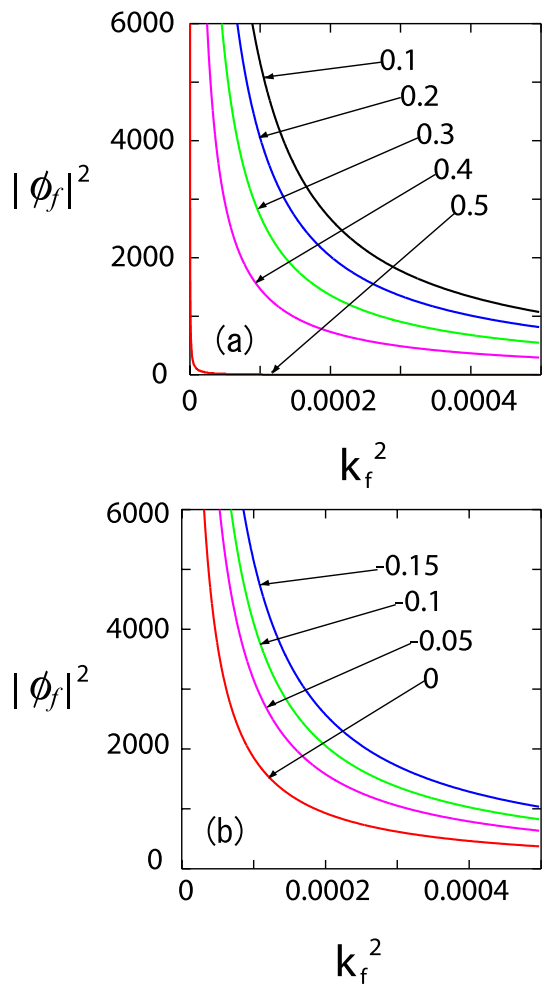

Fig. 3 Contour plot of (a) growth rate $\gamma_{\mathrm{q}}$ and (b) real frequency $\Omega_{\mathrm{q}}$ as a function of wave number $k_{\mathrm{f}}$ and mean flow energy $\left|\phi_{\mathrm{f}}\right|^{2}$. The real frequency of the mean flow is chosen as $\omega_{\mathrm{f}}=0$.

present case.

Next, we consider a time-varying mean flow by choosing $\omega_{\mathrm{f}} \neq 0$. The growth rate (a) $\gamma_{\mathrm{q}}$ and (b) real frequency $\Omega_{\mathrm{q}}$ are shown as a function of the frequency of mean flow $\omega_{\mathrm{f}}$. Here, the same wave number and mean flow energy as those used in Fig. 2 are chosen. The growth rate is found to increase with $\omega_{\mathrm{f}}$ as shown in Fig. 4 (a). Namely, the suppression effect is weakened by the time variation of the mean flow. However, the effect is found to be small in the region of ITG turbulence-driven zonal flow, i.e., $\omega_{\mathrm{f}}<0.1$, showing that the time variation of mean flow has less effect on the modulational process.

Here, we investigate the mechanism of the suppression of zonal flow generation due to a mean flow. For the sake of simplicity, the mean flow is assumed to be stationary, i.e., $\omega_{\mathrm{f}}=0$. As discussed above, the mean flow generates secondary side bands $k_{x+} \pm k_{\mathrm{f}}$ to the side band of $k_{x 0}$, i.e., $k_{x+}=k_{x 0}+k_{x \mathrm{q}}$. Here let us consider a sub-system consisting of a set of three side bands, i.e., $\phi_{k_{x+}}$ and $\phi_{k_{x+} \pm k_{\mathrm{f}}}$, and the mean flow $\phi_{k_{\mathrm{f}}}$, and construct a dispersion relation for the local modulational process by regarding that the mean flow $\phi_{k_{\mathrm{f}}}$ as a pump wave and a side band $\phi_{k_{x+}}$ as a seed. Then, the dispersion relation is written as follows:

$$
\begin{aligned}
& =\frac{\omega\left(1+k_{\mathrm{qs}}^{2}\right)+k_{y 0}}{2 k_{\mathrm{f}}^{2} k_{\mathrm{qs}}^{2} k_{y 0}^{2}\left(k_{\mathrm{qs}}^{2}-k_{\mathrm{f}}^{2}\right) \omega\left(\Lambda+k_{y 0} / \omega-4 k_{\mathrm{f}}^{2} k_{x+}^{2} / k_{\mathrm{qs}}^{2}\right)\left|\phi_{\mathrm{f}}\right|^{2}} \\
& {\left[\Lambda \omega+k_{y 0}\right]^{2}-4 k_{\mathrm{f}}^{2} k_{x+}^{2} \omega^{2}}
\end{aligned}
$$



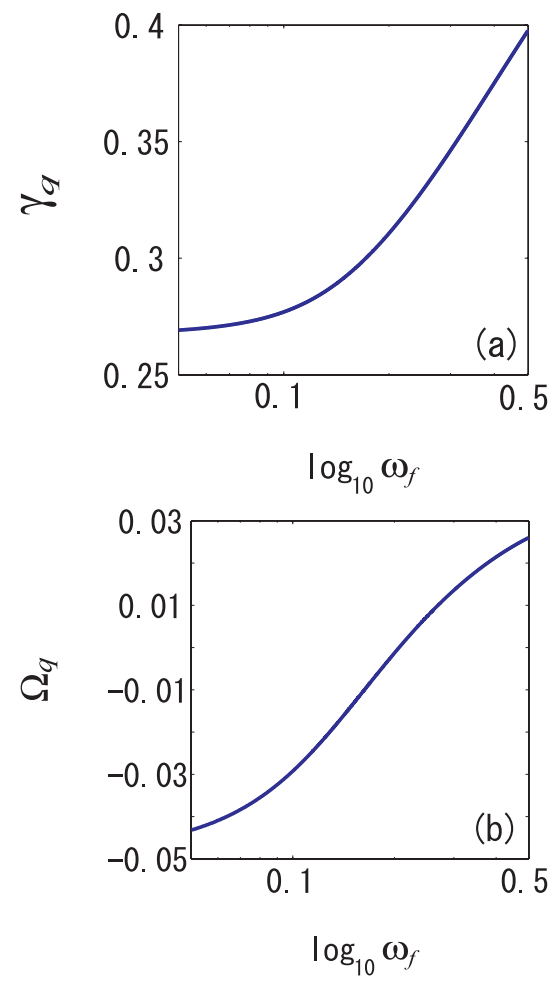

Fig. 4 The dependence of growth rate (a) $\gamma_{\mathrm{q}}$ and (b) real frequency $\Omega_{\mathrm{q}}$ as a function of real frequency $\omega_{\mathrm{f}}$. The wave number and mean flow energy are chosen as $k_{\mathrm{f}}=$ $0.0125,\left|\phi_{\mathrm{f}}\right|^{2}=2000$.

where definitions $\Lambda=1+k_{\mathrm{f}}^{2}+k_{\mathrm{qs}}^{2}$ and $k_{\mathrm{qs}}^{2}=k_{x+}^{2}+k_{y 0}^{2}$ are used. This is a third-order algebraic equation with respect to the complex frequency $\omega$ of the original side band $\phi_{k_{x+}}$. Therefore, when the root of Eq. (8) becomes complex conjugate solutions, i.e., $\omega=\omega_{r} \pm \mathrm{i} \gamma$, the modulational loop becomes unstable. In this case, the mean flow becomes a free energy source destabilizing the side band so that the original zonal flow instability may be enhanced. We have solved Eq. (8) in the same parameter region as seen in Fig. 3. Figure 5 illustrates the dependence of three roots of Eq. (8) as a function of $\left|\phi_{\mathrm{f}}\right|^{2}$ in the case of $k_{\mathrm{f}}=0.0125$. All three roots are found to be real values in the present parameter region, suggesting that the side band $\phi_{k_{x+}}$ exhibits a stationary oscillation without growing. Since the effect of the mean flow vanishes at $\left|\phi_{\mathrm{f}}\right|^{2}=0$, we choose a solution marked (*) in Fig. 5 that tends to $\omega=0$ at $\left|\phi_{\mathrm{f}}\right|^{2}=0$. The real frequency $|\omega|$ of the present sub-system in Fig. 5 is the same order as the change of the real frequency $\left|\Omega_{\mathrm{q}}\right|$ seen in Fig. 2 (b), i.e., for example $\left|\Omega_{\mathrm{q}}\right| \simeq 0.1$ and $|\omega| \simeq 0.4$ for $\left|\phi_{\mathrm{f}}\right|^{2}=2000$. Therefore, it may be noted that the stationary forced oscillation to the side band $k_{x \pm}=k_{x 0} \pm k_{x \mathrm{q}}$ due to the mean flow is related to the increase of $\left|\Omega_{\mathrm{q}}\right|$ with respect to $\left|\phi_{\mathrm{f}}\right|^{2}$ and the resultant reduction/stabilization of

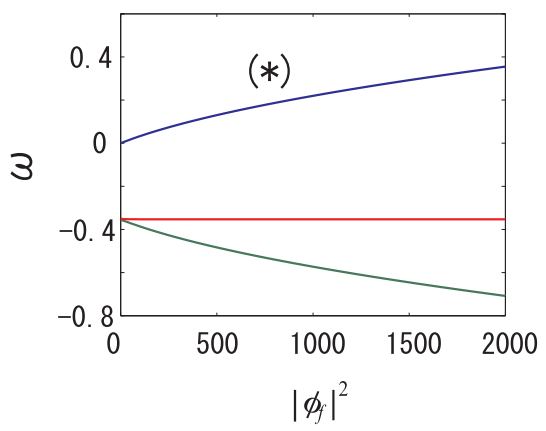

Fig. 5 The dependence of three roots of Eq. (8) as a function of mean flow energy $\left|\phi_{\mathrm{f}}\right|^{2}$. The wave number of the mean flow is chosen $k_{\mathrm{f}}=0.0125$.

zonal flow instability.

We have theoretically investigated the effects of an external mean flow on the generation of zonal flow due to ETG-driven turbulence in terms of a modulational instability analysis including a strong shearing regime. The main result demonstrates that the mean flow suppresses the growth rate of the zonal flow by having a large real frequency according to a relation $\gamma_{\mathrm{q}}=\gamma_{\mathrm{q} 0}\left(1-\alpha\left|\phi_{\mathrm{f}}\right|^{2} k_{\mathrm{f}}^{2}\right)$. The effect of the time-varying nature of the mean flow is found to be small in the frequency range of the GAM. This may be compared with the consideration that the suppression effect of turbulence is weakened in the presence of a time-varying $\boldsymbol{E} \times \boldsymbol{B}$ mean flow [10]. In our next paper, the present analysis will be extended to the toroidal geometry.

We would like to acknowledge Dr. J. Anderson for his useful discussions. This study was supported by a Grantin-Aid from the Japan Society for the Promotion of Science (No.15560721).

[1] P.H. Diamond, S.-I. Itoh, K. Itoh and T.S. Hahm, Plasma Phys. Control. Fusion 47, R35 (2005).

[2] A. Hasegawa and M. Wakatani, Phys. Rev. Lett. 59, 1581 (1987).

[3] L. Chen, Z. Lin and R. White, Phys. Plasmas 7, 3129 (2000).

[4] J.Q. Li and Y. Kishimoto, Phys. Plasmas 12, 054505 (2005).

[5] N. Miyato, Y. Kishimoto and J.Q. Li, Phys. Plasmas 11, 5557 (2004).

[6] T.S. Hahm and K.H. Burrell, Phys. Plasmas 3, 427 (1996).

[7] Y. Kishimoto, J.Y. Kim, W. Horton, T. Tajima, M.J. LeBrun and H. Shirai, Plasma Phys. Control. Fusion 41, A663 (1999).

[8] E. Kim and P. Diamond, Phys. Plasmas 10, 1698 (2003).

[9] A. Hasegawa and K. Mima, Phys. Fluids 21, 87 (1978).

[10] T.S. Hahm, M.A. Beer, Z. Lin, G.W. Hammett, W.W. Lee, and W.M. Tang, Phys. Plasmas 6, 922 (1999). 